\title{
A New Optimized Hybrid Local Lifting Wavelet Co-occurrence Texture Pattern for Content Based Medical Image Retrieval
}

\author{
https://doi.org/10.3991/ijoe.v17i11.25351 \\ R. Varaprasada Rao ${ }^{1(\varpi)}$, T. Jaya Chandra Prasad ${ }^{2}$ \\ ${ }^{1}$ Jawaharlal Nehru Technological University Anantapur, Andhra Pradesh, India \\ ${ }^{2}$ Rajeev Gandhi Memorial College of Engineering and Technology, Andhra Pradesh, India \\ rvr712@gmail.com
}

\begin{abstract}
Medical image retrieval (MIR) is a hard task owing to the varied patterns and structures in the medical images. The feature descriptors have been used to describe the images in most MIR approaches. Based on the local relationship, several feature descriptors of neighbouring image pixels have been proposed for MIR so far, but their low performance scores make them unsuitable. In this paper, an efficient optimized hybrid local lifting wavelet co-occurrence texture pattern for content-based MIR is proposed. Initially, image resize and Adaptive histogram equalization technique is used to carried out for contrast enhancement. Then Local Lifting Wavelet Co-occurrence Texture Pattern is derived using Local tetra pattern, Gradient directional pattern, lifting wavelet transform and Gray level co-occurrence matrix. An Equilibrium optimization technique is employed to select the most important features of an image from the obtained feature vectors (FV). Finally, to match the query image with the database images, distance between their FV is computed and the minimum distance images are considered as retrieval outcome. Three benchmark medical databases of various modalities (CT and MRI) are used to test the efficiency of the proposed method: EXACT-09, TCIA-CT, and OASIS. The experimental results prove that the proposed approach outperforms existing descriptors in terms of APR and ARR.
\end{abstract}

Keywords-medical image retrieval, local tetra pattern, gradient directional pattern, lifting wavelet transform, equilibrium optimization

\section{$1 \quad$ Introduction}

Computer assisted medical image analysis techniques help the medical doctors to make better their ability in disease judgment $[1,2]$. Medical image data is growing every day as a result of computerized clinical exams. The medical images are of various modalities such as X-ray, computer tomography (CT) and etc. Due to the large size of medical repositories and different modalities, image search or retrieval from the databases for a given query image has become a difficult task. Therefore, Content based medical image retrieval (CBMIR) was presented to solve these issues [3]. The CBMIR is an automatic image search technique unlike traditional image search methods such 
as manual search by the medical experts and text-based image search (TBIS). The manual search and TBIS requires huge man power and lead to blunders. In CBMIR, Image search can be done by their visual contents. The contents represent the characteristics of the image such as shape, colour, textures, edges, visual features and etc. In CBMIR, feature representation plays a crucial role to determine the image similarity. The local binary pattern (LBP) was once widely used to represent the image [4]. Due to its enormous popularity and simplicity, several LBP variants have been proposed for solving image retrieval problems in recent decades [5].

This paper proposes a new algorithm for efficient CBMIR and organized as follows. Section - 2 provides the literature survey; section -3 gives the explanation of proposed method; section - 4 provides the experimental results; and finally, section-5 concludes.

\section{Literature review}

Here, the most important feature descriptors and feature selection (FS) methods relate to the proposed approach are explained briefly.

\subsection{Feature descriptors for CBIR}

S. Murala et al. introduced a Directional Binary wavelet patterns (DBWP) [6] for MIR. The multi-resolution binary images were extracted by dividing the 8 -bit grayscale image into 8-bit binary planes. To extract the LBP features, binary wavelet transforms (BWT) was applied to every pixel of a plane and the DBWP was shaped in four directions for all BWT sub-bands. However, it increases the feature vector length. The local tetra pattern (LTrP) [7] was proposed as a way to use 1st-order derivatives in the horizontal and vertical directions to encode the relationship between the referenced pixel and its neighbours. Furthermore, Local ternary co-occurrence patterns (LTCoPs) [8] and Local mesh peak valley edge pattern (LMePVEP) [9] were proposed for CT MIR. The (n-1) $)^{\text {th }}$-order vertical and horizontal derivatives are combined with the Gabor transform to compute the $\mathrm{n}^{\text {th }}$-order LTrP. The LTCoP encoded the co-occurrence of identical ternary edges that were computed from the grey values of the centre and its neighbouring pixels. LMePVEP extracts the gray scale information like LTCoP but $1^{\text {st }}$ order derivative was used to derive the relations among the peak/valley edges. Anil Balaji Gonde et al. proposed 3D local transform pattern (3D-LTraP) [13] for image retrieval. To extract the features, spherical symmetric 3-dimensional local ternary pattern (SS3D-LTP) is used. The efficiency of SS-3D-LTP is determined by the threshold value and used to calculate ternary patterns. In addition, this method lacks multiscale and color information.

Local bit-plane dissimilarity pattern (LBDISP) [10] was introduced by S. R. Dubey et al. for CT MIR. Over each bit-plane, a dissimilarity map was found between the centre pixel and its neighbours, which was then encoded to form LBDISP. Local diagonal extrema pattern (LDEP) [11] was presented for CT MIR. The $1^{\text {st }}$ - order derivatives of local diagonals were used to find the values and indexes of the LDEs in order to manipulate the relationship between the any centre pixel and its diagonal neighbours of 
the image. The intensity values of the LDEs and the centre pixel were compared with indexes to make use of the relationship between the centre pixel and its neighbours. The feature vector's dimension is greatly reduced when only diagonal neighbours are considered, which speeds up image retrieval. Local wavelet pattern (LWP) [12] was proposed for CT MIR. The LWP was calculated for each pixel in the image using the relationship between the pixel and the local neighbouring data. Vandana kate et al. convolutional Deep-Net Model based on the extraction of random patches and enforcing depth-wise convolutions is proposed for training and classification of widely known benchmark Breast Cancer histopathology images. The classification result of these randomly extracted patches (size 50X50X3) is aggregated using majority vote casting in deciding the final image classification type [41]. Archana Kalidindi et al. presented a model using classification algorithms based on deep learning which can be used to classify or detect hemorrhage in a CT image [42].

\section{$2.2 \quad$ Feature selection}

There are two types of FS in the literature: filter and wrapper. The filter approaches point out the relevant features independently of the learning model. These approaches rank the attributes using the properties of the data and remove all features that do not perceive an adequate score $[16,17]$. The wrapper approaches rank the features using a pre-determined learning model, which can select the feature sub-set with a high evaluation measure [20]. Although the filter is computationally less expensive, the wrapper FS can often produce better results.

Kaur et. al. proposed a parameter-free bat algorithm to find an optimal set of features when classifying brain tumor MR images. The significant features were selected by minimizing the weighted distance between different categories. Li Zhang et al. [15] integrated the chaotic attractiveness movement, simulated annealing, and scattering strategies into the firefly algorithm for FS. Emary, Zawbaa, and Hassanien [18] developed a novel binary gray wolf optimization for dimensionality reduction. In this approach, a modified sigmoid function was implemented, and it enabled the wolves to conduct the search around the binary feature space.

\section{Proposed method}

The proposed optimized hybrid local lifting wavelet co-occurrence texture pattern for CBMIR block diagram is shown in Figure 1, which contains of pre-processing, feature extraction (FE), FS and similarity measurement and indexing modules.

In the pre-processing all the images are resized to [512 x 512] then Adaptive histogram equalization (AHE) technique is employed to improve their contrast. The FE and FS are important modules of the proposed CBMIR technique. Here a new approach is used to extract the features of an image. The local features of an image are extracted by using LTrP and GDP operators. Then lifting wavelet transform (LWT) is applied to the histograms of extracted local patterns to obtain the wavelet coefficients. Then Gray Level Co-Occurrence matrix (GLCM) technique is carried out to extract the texture 
features of an image. The extracted feature vectors (FV) have high dimensionality lead to high computational cost and large storage space. An equilibrium optimization technique is employed to select the optimal features. Hence, the dimension of the FV is reduced. The optimal features are most important features of an image. In image retrieval process, the query image has to match with the database images and the most relevant images can be considered as a retrieval outcome. Similar process is used to extract the FV of query image. Here, two distance measurement techniques are used as in [34 and 9] and their average is considered to match the query image features with the database images features. After calculating the distance, all the images are indexed by sorting in descending order based on their distances. The topmost minimum distance images are considered as retrieval outcome. Each module in the block diagram is explained in detailed in the below sections.

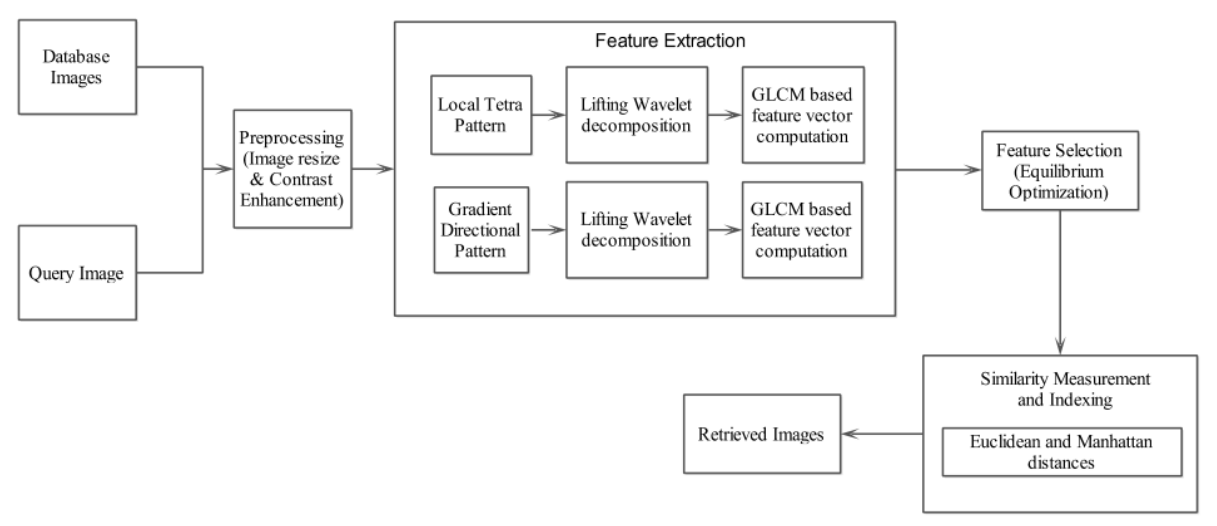

Fig. 1. Block diagram of proposed method

\subsection{Pre-processing}

During the pre-processing, all the images are first resized to [512X512] before being improved the contrast with the AHE technique. The AHE technique is well-known for enhancing contrast in both normal and medical images. The approach in Histogram Equalization (HE) entails applying to every pixel. In AHE, each pixel is modified based on adjacent pixels located in a region known as contextual region. The size of contextual region of an image with $n \times n$ pixels and $k$ intensity levels, is $m \times m$. Instead of using the histogram of neighbourhood pixels from a moving window, only the four closest grid points produce better results [21].

\subsection{Feature extraction}

In computer vision image contents or features are most important to represent an image. Various feature descriptors are discussed in the literature. The descriptors used in the proposed method are discussed in detail in this section. 
Local Tetra Pattern (LTrP). The LTrPs is introduced by adopting a key idea of local patterns LBP, Local derivative pattern (LDP), and local ternary pattern (LTP) which are presented in [22], [23], and [24] respectively. The LTrP define the spatial relation based on direction of centre gray pixel $\left(g_{c}\right)$ to derive the local texture structures. The local patterns will encode images with only ' 2 ' or ' 3 ' distinct values as " 0 ," " 1 ," or " 0 ," "1," " 1 " respectively. On the other hand, LTrP can encode images with '4" distinct values and extracts more detailed information. The LTrP encodes the relationship by combining the $(n-1)^{\text {th }}$-order derivatives of the $0^{0}$ and $90^{0}$ to determine the direction of the $g_{c}$ and its neighbors [7]. For an image $(I)$, the $1^{\text {st }}$ - order derivatives along $0^{0}$ and $90^{\circ}$ are written as in eqns. (1) and (2). Let $g_{h}$ is horizontal and $g_{v}$ vertical neighbors of $g_{c}$.

$$
\begin{aligned}
& I_{0^{0}}^{1}\left(g_{c}\right)=I\left(g_{h}\right)-I\left(g_{c}\right) \\
& I_{90^{0}}^{1}\left(g_{c}\right)=I\left(g_{v}\right)-I\left(g_{c}\right)
\end{aligned}
$$

and the direction of the $g_{c}$ can be calculated as

$$
I_{\text {Dir }}^{1}\left(g_{c}\right)=\left\{\begin{array}{c}
1, I_{0^{0}}^{1}\left(g_{c}\right) \geq 0 \text { and } I_{90^{0}}^{1}\left(g_{c}\right) \geq 0 \\
2, I_{0^{0}}^{1}\left(g_{c}\right)<0 \text { and } I_{90^{0}}^{1}\left(g_{c}\right) \geq 0 \\
3, I_{0^{0}}^{1}\left(g_{c}\right)<0 \text { and } I_{90^{0}}^{1}\left(g_{c}\right)<0 \\
4, I_{0^{0}}^{1}\left(g_{c}\right) \geq 0 \text { and } I_{90^{0}}^{1}\left(g_{c}\right)<0
\end{array}\right.
$$

According to eqn. (3), the possible directions for each centre pixel are 1, 2, 3, or 4, and the image is ultimately translated into ' 4 '-values. The $2^{\text {nd }}$-order $\operatorname{Lrr} P^{2}\left(g_{c}\right)$ is represented as

$$
\begin{gathered}
\quad \operatorname{Tr}^{2}\left(g_{c}\right)=\left\{f_{3}\left(I_{\text {Dir }}^{1}\left(g_{c}\right), I_{\text {Dir }}^{1}\left(g_{1}\right)\right), f_{3}\left(I_{\text {Dir }}^{1}\left(g_{c}\right), I_{\text {Dir }}^{1}\left(g_{2}\right)\right),\right. \\
\left.\ldots . f_{3}\left(I_{\text {Dir }}^{1}\left(g_{c}\right), I_{\text {Dir }}^{1}\left(g_{p}\right)\right)\right\}\left.\right|_{p=8} \\
f_{3}\left(I_{\text {Dir }}^{1}\left(g_{c}\right), I_{\text {Dir }}^{1}\left(g_{p}\right)=\left\{\begin{array}{c}
0, I_{\text {Dir }}^{1}\left(g_{c}\right)=I_{\text {Dir }}^{1}\left(g_{p}\right) \\
I_{\text {Dir }}^{1}\left(g_{p}\right), \text { elsewhere }
\end{array}\right.\right.
\end{gathered}
$$

The 8-bit tetra pattern is calculated for each $g_{c}$ from eqns. (4) and (5). Then, all the patterns are divided into ' 4 ' parts based on the $g_{c}$ direction. Finally, ' 3 ' binary patterns are converted from the tetra patterns for each direction.

where $I_{\text {Dir }}^{1}\left(g_{c}\right)$ denotes the direction of $g_{c}$ and obtained using eqn. (3) be ' 1 '; then, $\operatorname{LTr} P^{2}$ can be represented by separating it into 3 binary patterns as follows:

$$
\begin{gathered}
\left.L \operatorname{Tr} P^{2}\right|_{\text {Direction }=2,3,4}=\sum_{p=1}^{P} 2^{(p-1)} \times\left. f_{4}\left(\operatorname{LTr} P^{2}\left(g_{c}\right)\right)\right|_{\text {Direction }=2,3,4} \\
\left.f_{4}\left(\operatorname{LTr} P^{2}\left(g_{c}\right)\right)\right|_{\text {Direction }=\varnothing}= \begin{cases}1, \text { if } & \operatorname{Lr} P^{2}\left(g_{c}\right)=\emptyset \\
0, & \text { otherwise }\end{cases}
\end{gathered}
$$


Where $\emptyset=2,3,4$, and ' $P$ ' denoted as the number of neighbors of the $g_{c}$. In the same way, the remaining three tetra patterns (parts) of the $g_{c}$ are transformed to $12(4 \times 3)$ binary patterns. The $13^{\text {th }}$ binary pattern (magnitude) is calculated via eqn. (9).

$$
\begin{gathered}
M_{I^{1}}\left(g_{p}\right)=\sqrt{\left(\left(I_{0^{0}}^{1}\left(g_{c}\right)\right)^{2}+\left(I_{0^{0}}^{1}\left(g_{c}\right)\right)^{2}\right)} \\
\mathrm{LP}=\sum_{p=1}^{P} 2^{(p-1)} \times\left. f_{1}\left(M_{I^{1}}\left(g_{p}\right)-M_{I^{1}}\left(g_{c}\right)\right)\right|_{P=8}
\end{gathered}
$$

For the local pattern, $2^{P}$ combinations of LBPs are possible with $P$ neighborhoods which results the length of feature vector is $2^{P}$. The length of the feature vector is leads to the high computational cost and it can be reduced by considering uniform pattern [25]. The uniform pattern consists less number of discontinuities which are less than two in the circular binary representation (CBR), whereas the nonuniform patterns are those with more than two discontinuities. Therefore, for an input image, the distinct uniform patterns would be $\mathrm{P}(\mathrm{P}-1)+2$. The histogram is constructed for the whole image using PTN as in eqn. (10).

$$
\begin{gathered}
H_{s}(l)=\frac{1}{N_{1} \times N_{2}} \sum_{j=1}^{N_{1}} \sum_{k=1}^{N_{2}} f_{5}(P T N(j, k), l: l \in[0, P(P-1)+2] \\
f_{5}(x, y)=\left\{\begin{array}{cc}
1, & \text { if } x=y \\
0, & \text { else }
\end{array}\right.
\end{gathered}
$$

where $N_{1} \times N_{2}$ is the size of the input image.

Gradient Directional Pattern (GDP). The LBP descriptor sets the threshold to the value of the centre pixel $\left(g_{c}\right)$. As a result, LBP patterns are sensitive to interference to noise and illumination variations, as even minor changes will cause their value to change in relation to the $g_{c}$. Although some recent approaches for forming binary patterns used more stable gradient magnitudes and edge response values instead of gray level [26]. The gradient direction angles (GDA) of the pixels are quantized to form a local texture pattern. The gradient vector's direction of each pixel in an image is determined as in eqn. (12).

$$
\alpha(x, y)=\tan ^{-1}\left(\frac{G_{y}}{G_{x}}\right)
$$

Where $\alpha(x, y)$ denotes the GDA of the pixel (x,y), and $G_{x}$ and $G_{y}$ are the elements of two gradient vectors. The Sobel operator is convolved with horizontal and vertical kernel of the image to get the values of $G_{x}$ and $G_{y}$. Then GDP descriptor takes a $3 \times 3$ neighbourhood pixels around each pixel of the image using obtained GDA as in eqn. (13). A threshold $t$ (user specified) is used to quantize the neighbouring GDA with respect to the pixel's direction angle. Here, neighbours with a gradient angle $\pm t$ about the centre angle are quantised as in eqn. (14).

$$
\begin{gathered}
G D P\left(x_{c}, y_{c}\right)=\sum_{p=0}^{P-1} s\left(G D_{p}, G D_{c}\right) 2^{p} \\
s\left(G D_{p}, G D_{c}\right)=\left\{\begin{array}{lr}
1, & G D_{c}-t \leq G D_{p} \leq G D_{c}+t \\
0, & \text { otherwise }
\end{array}\right.
\end{gathered}
$$


Where $G D_{c}$ and $G D_{p}$ are denoted as GDA of the pixel $\left(x_{c}, y_{c}\right)$ and GDP angles of its neighbours respectively. The GDP histogram is represented as,

$$
\begin{aligned}
& H_{G D P}(i)=\sum_{x=1}^{M} \sum_{y=1}^{N} f(G D P(x, y), i) \\
& \text { Where } f(a, i)=\left\{\begin{array}{lr}
1, & a=i \\
0, & \text { otherwise }
\end{array}\right.
\end{aligned}
$$

Lifting wavelet decomposition of LTrP and GDP histograms. The eqns. 10 and 15 of LTrP and GDP histograms represents the same whole 2D image individually. To derive the lifting wavelet coefficients, LWT is applied to LTrP and GDP histograms. Basically, lifting wavelet decomposition can be divided into 3 steps such as (i) split, (ii) predict, and (iii) update.

(i) Split: Initially, the original signal $F_{0}$ is split into two subsets as even $\left(F_{1}\right)$ and odd $\left(D_{1}\right)$ sequences using a simple wavelet known as lazy wavelet. It is represented in eqn. (17).

$$
\operatorname{Split}\left(F_{o}\right)=\left(\text { Even }_{1}, \operatorname{Odd}_{1}\right)=\left(F_{1}, D_{1}\right)
$$

Generally, $F_{1}$ and $D_{1}$ denotes the LF approximate component and the HF detail component respectively.

(ii) Predict: In this step, the even sequence $F_{1}$ is used to predict the odd sequence $D_{1}$ based on the correlation in the original signal. The prediction can be expressed as:

$$
D^{\prime}{ }_{1}=D_{1}-P\left(F_{1}\right)
$$

(iii) Update: Here, the modified odd sequence $U\left(D_{1}\right)$ is added to the even sequence $F_{1}$ to form the updated even sequence. The updated even sequence is expressed in eqn. (19):

$$
F^{\prime}{ }_{1}=F_{1}+U\left(D_{1}\right)
$$

Similarly, the 3 steps can be used to split the data subset $F_{1}$ into $D_{2}$ and $F_{2}$. The integer wavelet is decomposed the original data set $F_{0}$ as $\left\{F_{n}, D_{n}, D_{n-1}, \ldots \ldots, D_{1}\right\}$, where $F_{n}$ denotes the low frequency (LF) part of signals, and $\left\{D_{n}, D_{n-1}, \ldots \ldots, D_{1}\right\}$ is the high frequency (HF) part of signals. From the above steps, the lifting scheme is realized in situ operations. These steps are repeatedly used in lifting filter banks to obtain wavelet transform coefficients.

GLCM based feature vector computation. The GLCM is a statistical method for analyzing textures in a grayscale image [27]. Let $I(k, k)$ will be the neighborhood grayscale image of a central pixel $\left(n_{c}, m_{c}\right)$. The cooccurrence values at a given distance from a specified pixel $\left(n_{c}, m_{c}\right)$ is distributed to calculate the cooccurrence matrix of the image. The cooccurrence matrix $C_{M}=C_{\left(d_{x}, d_{y}\right)}(k, k)$ of the image $I(k, k)$ is defined as

$$
C_{M}=\sum_{n=1}^{k} \sum_{m=1}^{k}\left\{\begin{array}{lr}
1, & \text { if } I(m, n)=k, I\left(n+d_{x}\right)=k \\
0, & \text { otherwise }
\end{array}\right.
$$

Where $\left(d_{x}, d_{y}\right)$ are defined as 


$$
d_{x}=d \cdot \cos (\theta), d_{x}=d \cdot \sin (\theta)
$$

Where $\theta$ and $d$ denotes the direction and distance from the central pixel $\left(n_{c}, m_{c}\right)$ respectively that is shown in Figure 2.

From the cooccurrence matrix $C_{M}=C_{\left(d_{x}, d_{y}\right)}(k, k)$ and for each $\theta$ angle, the correlation, the energy, and the homogeneity are computed using eqns. (22), (23), (24) and (25).

$$
\begin{gathered}
\theta_{f(\text { contrast })}=\sum_{i=1}^{L} \sum_{j=1}^{L}(i-j)^{2} C_{M}, \\
\theta_{f(\text { correlation })}=\sum_{i=1}^{L} \sum_{j=1}^{L} C_{M}\left[\frac{\left(i-\mu_{i}\right)\left(j-\mu_{j}\right)}{\sqrt{\left(\sigma_{i}^{2}\right)\left(\sigma_{j}^{2}\right)}}\right]
\end{gathered}
$$

Where $\mu_{i}=\sum_{i=1}^{L} \sum_{j=1}^{L} i C_{M}, \quad \mu_{j}=\sum_{i=1}^{L} \sum_{j=1}^{L} j C_{M}, \quad \sigma_{i}^{2}=\sum_{i=1}^{L} \sum_{j=1}^{L} C_{M}\left(i-\mu_{i}^{2}\right)$ and $\sigma_{j}^{2}=\sum_{i=1}^{L} \sum_{j=1}^{L} C_{M}\left(j-\mu_{j}^{2}\right)$.

$$
\begin{aligned}
\theta_{f(\text { energy })} & =\sum_{i=1}^{L} \sum_{j=1}^{L} C_{M}^{2} \\
& \theta_{f(\text { Homogeneity })}=\sum_{i=1}^{L} \sum_{j=1}^{L} \frac{C_{M}}{1+|i-j|}
\end{aligned}
$$

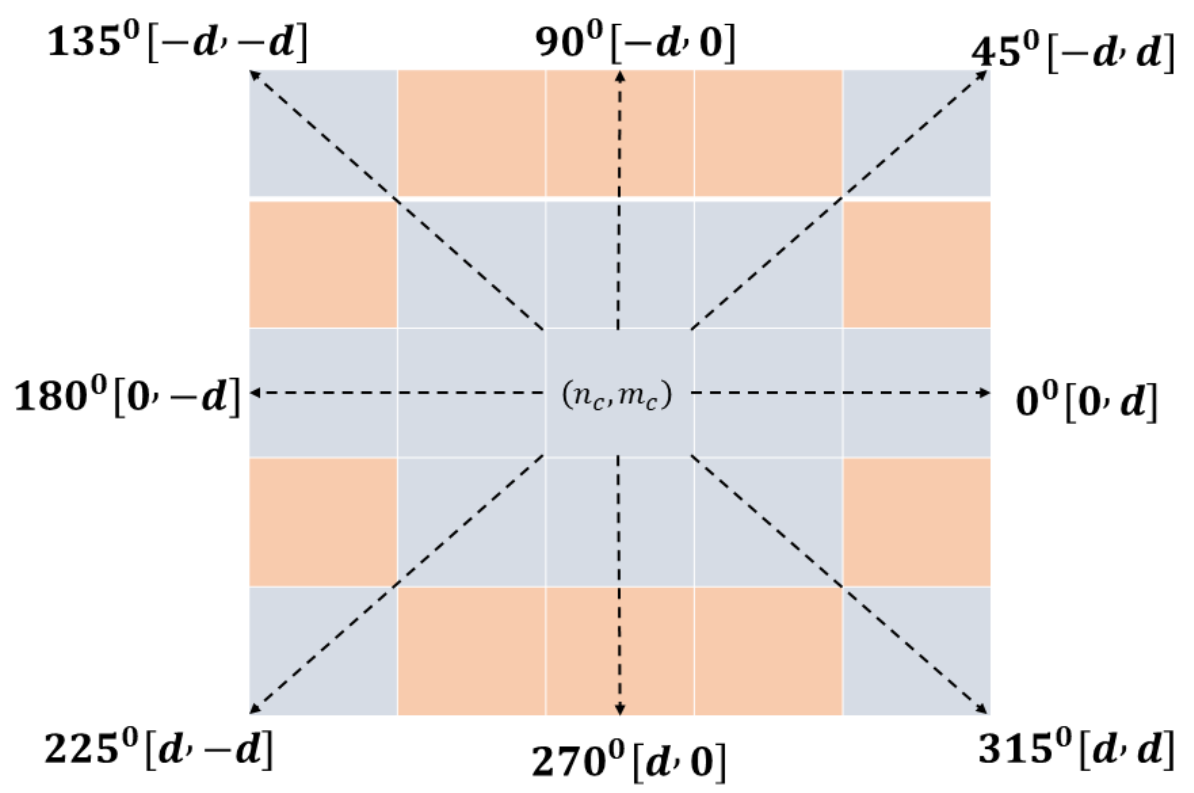

Fig. 2. The direction and distance relationships of the neighborhood pixels from the central pixel $\left(n_{c}, m_{c}\right)$ 


\subsection{Feature selection}

From the above steps, the obtained texture feature vectors suffered with high dimensionality. Conventionally immense amount of data, irrelevant and redundant features make the process complex may adversely worsen the processing accuracy. To remove the noisy information and define the most significant features, the feature selection (FS) process should be considered. Here Equilibrium optimizer (EO) is employed to select the most important features from the obtained FV.

Equilibrium optimization. The Equilibrium optimizer (EO) is motivated by the concept of dynamic source and sink models in measuring equilibrium states [19]. Like other metaheuristic algorithms, EO generates an initial population of stochastic solutions to start the optimization process. In EO, an initial population of $N$ particles is computed as follows:

$$
Y_{i}^{d}=Y_{\min }+r n d_{i}^{d i}\left(Y_{\max }-Y_{\min }\right) \text {, }
$$

Where $i=1,2,3, \ldots, N$ and $d i=1,2,3, \ldots, D$

Where $Y$ is the position of the particle, $N$ represents the number of particles, $D$ is the number of dimensions, and $r n d$ is a random vector between $[0,1]$. The $Y_{\text {max }}$ and $Y_{\text {min }}$ are the maximum and minimum dimension values. After generating the initial population, the particles are evaluated with a specific fitness function, and the equilibrium candidates were identified.

Equilibrium Pool and Candidates. In EO, there is an equilibrium pool to store promising candidates. Correspondingly, four best-so-far particles and their average are stored in the equilibrium pool and will be used for the updating process. These four best-so-far candidates can assist the EO to explore the untried areas, which ensures a high exploration. On the one hand, the average of these candidates can help to exploit the areas near the best solution to realize the global optimum. Following this line of thoughts, the equilibrium pool is constructed as follows:

$$
\begin{gathered}
Y_{e q, p o o l}=\left\{Y_{e q(1)}, Y_{e q(2)}, Y_{e q(3)}, Y_{e q(4)}, Y_{e q(a v g)}\right\} \\
Y_{e q(a v g)}=\frac{Y_{e q(1)}+Y_{e q(2)}+Y_{e q(3)}+Y_{e q(4)}+Y_{e q(a v g)}}{4}
\end{gathered}
$$

Where $Y_{e q, p o o l}$ is the equilibrium pool, $Y_{e q(1)}, Y_{e q(2)}, Y_{e q(3)}$, and $Y_{e q(4)}$ are the 4 best candidates and $Y_{e q(a v g)}$ is its average. In each iteration, the particles update their positions with random selection among these five candidates (same probability).

Exponential Term. The exponential term is an important factor that will help EO to retain a proper balance between global and local searches and defined in eqn. (29):

$$
F=e^{-\lambda\left(T-T_{0}\right)}
$$

where $\lambda$ is a random vector between $[0,1], T$ is the time that can be computed as in eqn. (30):

$$
T=\left(1-\frac{\text { Iter }}{\operatorname{Iter}(\operatorname{Max})}\right)\left(\alpha \frac{\text { Iter }}{\operatorname{Iter}(\operatorname{Max})}\right)
$$


where Iter is the current iteration, Iter $(M a x)$ is the maximum number of iterations, and $\alpha$ is a constant used to control the local search behavior. On the other hand, $T_{0}$ is a parameter used to manage exploration and exploitation as follows:

$$
T_{0}=\frac{1}{\lambda} \operatorname{In}\left(-\beta \operatorname{sign}(r v-0.5)\left[1-e^{-\lambda T}\right]\right)+T
$$

where $r v$ is a random vector between $[0,1]$, and $\beta$ is a constant that is used to control the exploration capacity. As given in Equation (30), the larger the value of $\beta$, the better the exploration capability. According to [19], $\alpha$ and $\beta$ are equal to 1 and 2 respectively. By substituting the eqn. (30) into eqn. (28), the final version of the exponential term can be redefined as below:

$$
F=\left(\beta \operatorname{sign}(r v-0.5)\left[e^{-\lambda T}-1\right]\right)
$$

Generation Rate. Another important factor in EO is the generation rate (GR), which helps to explore the search domain. In EO, the GR is formulated as follows:

$$
G=G_{0} e^{-\lambda\left(T-T_{0}\right)}=G_{0} F
$$

Where $G_{0}=G C P\left(Y_{e q}-\lambda Y\right)$

$$
G C P=\left\{\begin{array}{cc}
0.5 r v_{1}, & r v_{2} \geq G P \\
0, & r v_{2}<G P
\end{array}\right.
$$

Where $r v_{1}$ and $r v_{2}$ are two random vectors between [0,1], respectively. The GCP is the generation rate control parameter, and it is computed using eqn. (35). Eventually, the updating rule of $\mathrm{EO}$ is defined as:

$$
Y=Y_{e q}+\left(Y-Y_{e q}\right) F+\frac{G}{\lambda V}(1-F)
$$

where $F$ is the exponential term, $G$ is the generate rate, $Y_{e q}$ is a random candidate from equilibrium pool, and $V$ is a constant unit with a value equal to 1 [19].

Memory Saving. In EO, a mechanism resembles the pbest concept in particle swarm optimization is implemented. If the fitness value attained by the particle in the current iteration is better than the previous iteration, then the particle with better fitness will be saved and stored in pbest.

\subsection{Similarity measurement and indexing}

In CBMIR, the query image has to match with the database images. Finally, similarity is determined between the query and the database images based on the distance between their feature vectors. The distance is measured using eqn. (37) and eqn. (38) as in [28] and [7] respectively.

$$
\begin{aligned}
& d_{1}(a, b)=\sum_{i=1}^{n}\left|a_{i}-b_{i}\right| \\
& d_{2}(a, b)=\sum_{i=1}^{n} \frac{\left(a_{i}-b_{i}\right)}{1+\left(a_{i}+b_{i}\right)}
\end{aligned}
$$


The average of the distances $d_{1}$ and $d_{2}$ is represented in eqn. (39).

$$
d_{\text {avg }}=\text { Average }\left(d_{1}, d_{2}\right)
$$

Based on the distance between the images, they are sorted in descending order. The topmost minimum distance images are considered as retrieval outcome.

\section{$4 \quad$ Experimental results discussions}

The experimental outcomes are discussed in this section to assess the proposed approach's performance in various aspects. The performance of the proposed approach is evaluated by make use of precision $(\mathrm{P})$ and recall $(\mathrm{R})$ and are calculated as in eqns. (40) and (41).

$$
\begin{gathered}
P=\frac{\text { No. of relevent images retrieved }}{\text { No.of images retrieved }} \\
R=\frac{\text { No. of relevent images retrieved }}{\text { No.of relevent images in the database }}
\end{gathered}
$$

The eqns. (40) and (41) are calculated for each database image that is used as a query and compared with all database images. The average precision rate (APR), and average recall rate (ARR) are the mean average of the eqns. (40) and (41) respectively. The $F_{\text {value }}$ is computed based on the APR and ARR values as in eqn. (42).

$$
F_{\text {value }}=\frac{2 \times A R P \times A R R}{A R P+A R R}
$$

Three experiments are carried out on publicly accessible medical databases. The details of databases are briefly explained in Table 1 . The detailed explanation about databases and experimental setups can be seen in [12,36 and 37] and used here are identical.

Table 1. Illustration of image databases

\begin{tabular}{|l|c|c|c|c|}
\hline Database name & Modality & Total No. of images & No. of groups & No. of images in each group \\
\hline EXACT-09 [29] & CT & 675 & 19 & $20-55$ \\
\hline TCIA [30] & CT & 604 & 8 & $41-140$ \\
\hline OASIS [31] & MRI & 421 & 4 & $89-124$ \\
\hline
\end{tabular}

\subsection{Experimental results comparisons}

The results are compared with the existing feature descriptors such as LBP [4], LTP [32], LDP [33], LTrP [7], LTCoP [8], LMeP [34], SS-3D-LTP [35], LWP [12], Histogram of Compressed Scattering Coefficients (HCSC) [36], Directional binary wavelet pattern (DBWP) [6], Directional local ternary quantized extrema Pattern (DLTe-rQEP) [38], Local neighboring binary pattern (LNBP) [39], Fast discrete curvelet transform (FDCT) [40] and Local directional frequency encoded pattern (LDFEP) [37]. The performance comparisons of the proposed approach with existing descriptors on the 
EXACT09, TCIA CT and OASIS-MRI databases are presented in Table 2 and Table 3 respectively.

Over the EXACT09 dataset, the proposed approach attained ARR, APR, and Fvalue are $0.2808,0.9253$ and 0.4308 respectively. Among the all descriptors in the Table 2, LWP, HCSC and LDFEP are comparable because performed better than other descriptors. The proposed approach is improved by $11.48 \%, 1.12 \%$ and $3.97 \%$ in terms of APR compared with the LWP, HCSC and LDFEP respectively. One of the retrieval results (with $100 \%$ precision) for the input query is shown in Figure 3 (a).
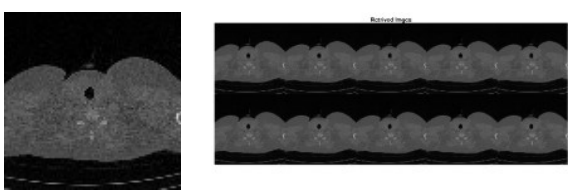

(a)
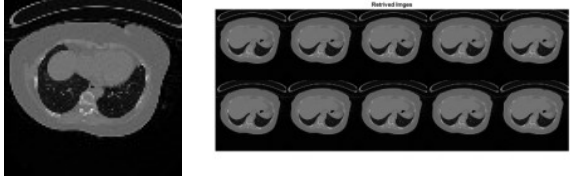

(b)

Fig. 3. Query Image and Top 10 retrieved images Retrieval result of (a) EXACT09 (b) TCIA CT

Table 2. Comparisons of different descriptors and proposed approach performances over EXACT09 and TCIA CT databases

\begin{tabular}{|l|c|c|c|c|c|c|}
\hline Database Name & \multicolumn{3}{|c|}{ EXACT09 } & \multicolumn{3}{c|}{ TCIA CT } \\
\hline Methods & $\boldsymbol{A P R}$ & $\boldsymbol{A R \boldsymbol { R }}$ & $\boldsymbol{F}_{\text {value }}$ & $\boldsymbol{A P R}$ & $\boldsymbol{A R R}$ & $\boldsymbol{F}_{\text {value }}$ \\
\hline LBP [4] & 0.6503 & 0.1951 & 0.3002 & 0.6691 & 0.0974 & 0.1700 \\
\hline LDP [33] & 0.5440 & 0.1619 & 0.2495 & 0.6906 & 0.1005 & 0.1755 \\
\hline LTrP [7] & 0.5782 & 0.1729 & 0.2662 & 0.7183 & 0.1033 & 0.1806 \\
\hline LTP [32] & 0.6209 & 0.1854 & 0.2855 & 0.7469 & 0.1095 & 0.1910 \\
\hline LTCoP [8] & 0.7348 & 0.2216 & 0.3405 & 0.7440 & 0.1092 & 0.1904 \\
\hline LMeP [34] & 0.6323 & 0.1891 & 0.2911 & 0.7371 & 0.1077 & 0.1879 \\
\hline SS_3D_LTP [35] & 0.6700 & 0.2009 & 0.3091 & 0.8054 & 0.1171 & 0.2045 \\
\hline LWP [12] & 0.8300 & 0.2487 & 0.3827 & 0.8840 & 0.1309 & 0.2280 \\
\hline HCSC [36] & 0.915 & 0.2883 & 0.4384 & 0.9512 & 0.1452 & 0.2520 \\
\hline LDFEP [37] & 0.8899 & 0.1916 & 0.3153 & - & - & - \\
\hline Proposed Method & 0.9253 & 0.2808 & 0.4308 & 0.9745 & 0.1453 & 0.2529 \\
\hline
\end{tabular}

Over the TCIA CT image data set LWP and HCSC are the best comparable descriptors in the Table 2. The proposed method obtained APR, ARR and $F_{\text {value }}$ as 0.9745 , 0.1453 and 0.2529 respectively. The proposed method is improved by $\{10.23 \%, 11 \%$ and $10.92 \%\},\{2.45 \%, 0.068 \%$ and $0.357 \%\}$ in terms of $\left\{A P R, A R R\right.$ and $\left.F_{\text {value }}\right\}$ in contrast to LWP and HCSC methods respectively. One of the retrieval results (query and retrieved top 10 images i.e., 100\% precision) is shown in Figure 3 (b).

The performances of different methods in terms of APR, ARR, and $F_{\text {value }}$ over the OASIS-MRI database (at No. of best matches $=10$ ) is illustrated in Table 3. One of the retrieval results is shown in Figure 4. 

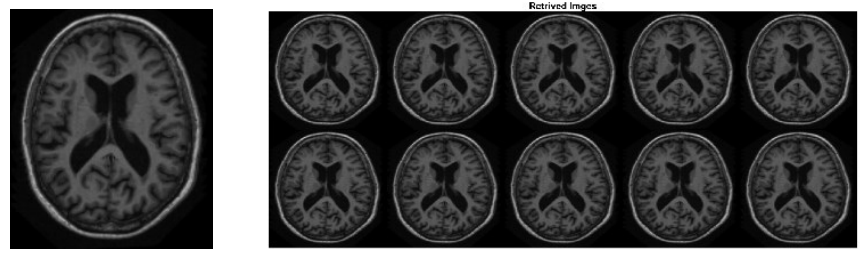

Fig. 4. Retrieval result of OASIS MRI database: Query image and Top 10 retrieved images

The performance comparison of the proposed method in terms of APR, ARR and $\mathrm{F}_{\text {value }}$ over the OASIS MRI database and presented in the Table 3. The proposed method attained $92.16 \%, 8.75 \%$ and $15.98 \%$ in terms of APR, ARR and $F_{\text {value }}$ and improved by $5.48 \%, 24.11 \%$ and 22.64 in contrast to LDFEP respectively.

Table 3. Comparison of different descriptors and proposed approach performances over OASIS MRI database

\begin{tabular}{|l|c|c|c|c|c|c|c|c|}
\hline \multicolumn{10}{|c|}{ OASIS MRI } \\
\hline Methods & $\begin{array}{c}\text { LBP } \\
{[4]}\end{array}$ & $\begin{array}{c}\text { DBWP } \\
{[\mathbf{6}]}\end{array}$ & $\begin{array}{c}\text { LMeP } \\
{[34]}\end{array}$ & $\begin{array}{c}\text { DLTe-rQEP } \\
{[38]}\end{array}$ & $\begin{array}{c}\text { LNBP } \\
{[39]}\end{array}$ & $\begin{array}{c}\text { FDCT } \\
{[\mathbf{3 0 ]}}\end{array}$ & $\begin{array}{c}\text { LDFEP } \\
{[37]}\end{array}$ & $\begin{array}{c}\text { Proposed } \\
\text { Method }\end{array}$ \\
\hline $\mathrm{APR}$ & 42.63 & 47.05 & 44.96 & 45.10 & 51.63 & 51.55 & 87.37 & 92.16 \\
\hline $\mathrm{ARR}$ & 2.21 & 3.89 & 2.74 & 2.82 & 5.57 & 4.76 & 7.04 & 8.75 \\
\hline $\mathrm{F}_{\text {value }}$ & 4.202 & 7.185 & 5.165 & 5.308 & 10.055 & 8.715 & 13.030 & 15.98 \\
\hline
\end{tabular}

The comparison of proposed method performance in terms of APR (\%) in contrast with existing descriptors over EXACT09, TCIA CT, and OASIS MRI databases with varying top matches are shown in Figure $5(\mathrm{a}-\mathrm{c})$. The proposed method outperformed in each database compared to existing descriptors. From the experimental results it is evidenced that the proposed technique is performed superior than the existing methods over three databases. 


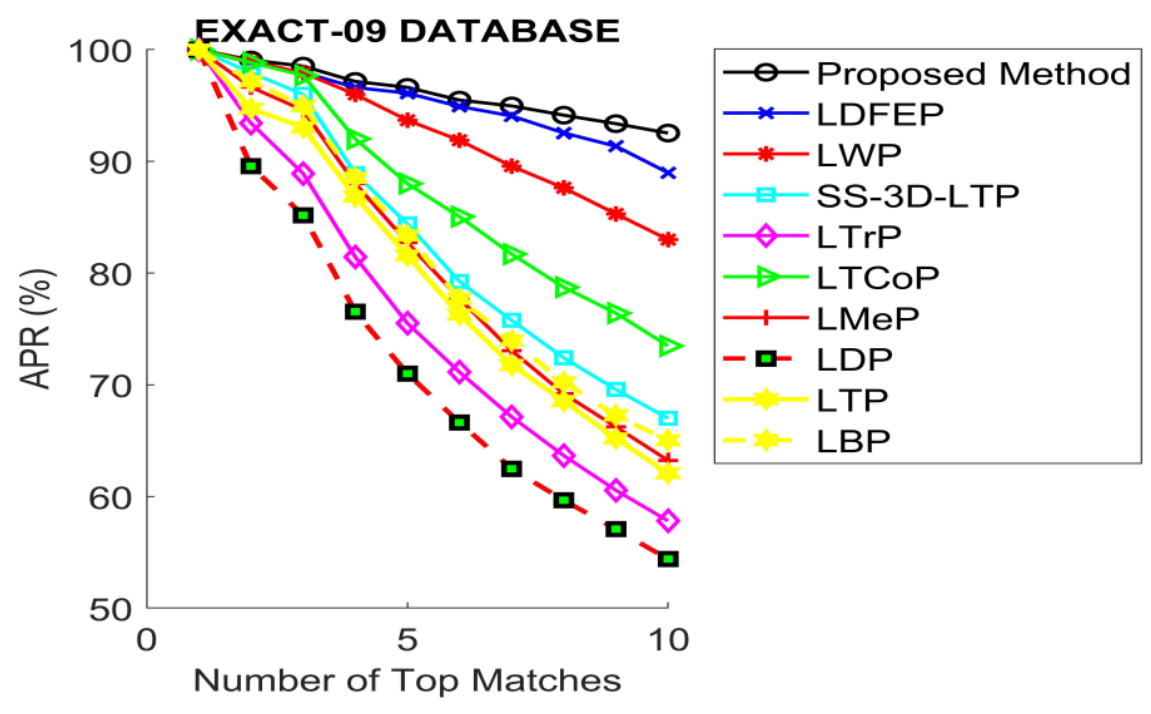

(a)

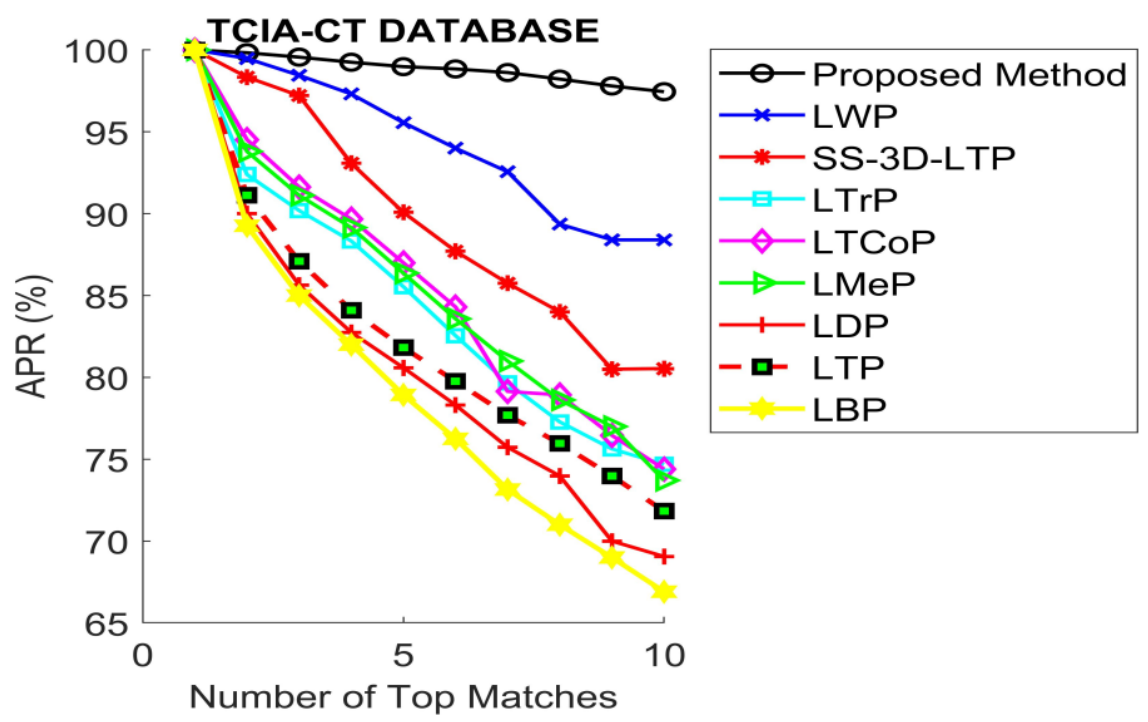

(b) 


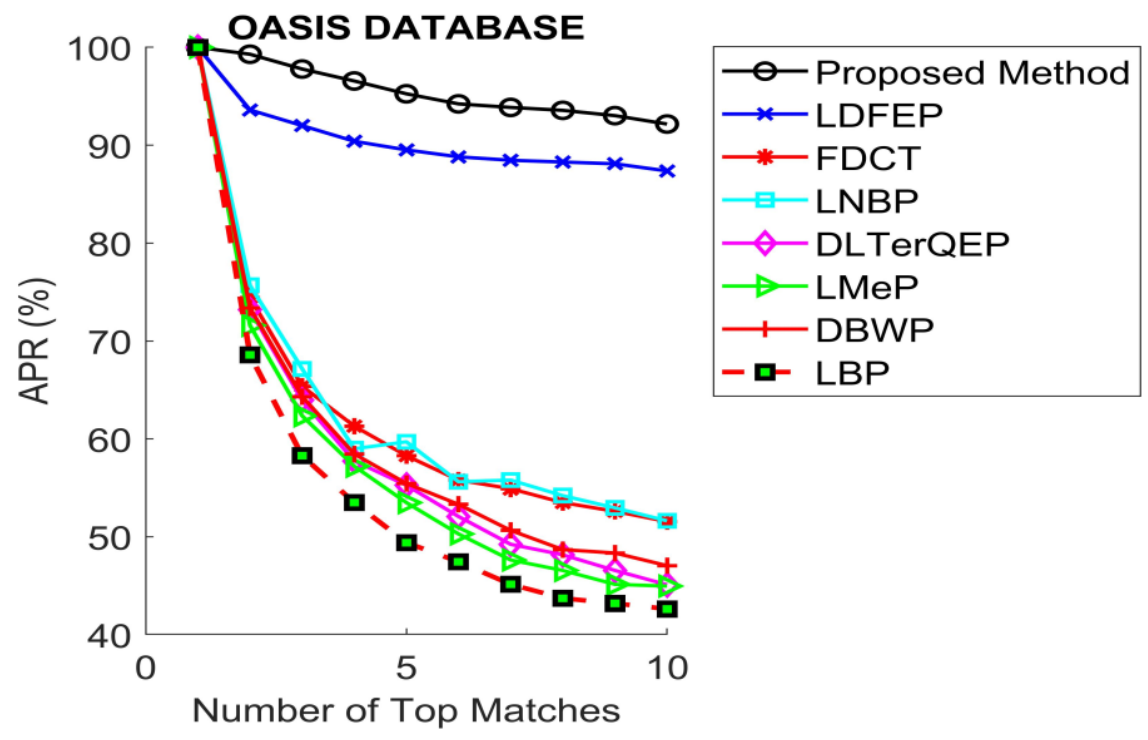

(c)

Fig. 5. The comparison of retrieval performance in terms of APR (\%) Vs No. of top matches over (a) EXACT09 (b) TCIA CT and (c) OASIS MRI databases

\section{Conclusions}

In this paper a new approach, an equilibrium optimized hybrid local lifting wavelet co-occurrence texture pattern for CBMIR is proposed. The lifting wavelet decomposition is performed on histograms of local tetra and gradient directional patterns. Then GLCM is computed for co-occurrence of the neighbouring pixels with the central pixels. Then an Equilibrium optimization technique is carried out to condense the FV dimension and to select the most important features of an image. Finally, the distance is calculated between the optimised query and the database image FV. All the images are arranged in descending order based on their distances. The top most minimum distance images are considered as retrieval outcome. To test the retrieval performance of the proposed method, 3 tests are carried out on two CT and one MRI databases namely, EXACT09, TCIA and OASIS respectively. The proposed method's performance is evaluated in terms of APR, ARR and $\mathrm{F}_{\text {value }}$ and compared with the existing descriptors. The experimental results proved that the proposed method is performed better in each database and it can be used to diagnose CT and MRI medical images effectively. 


\section{References}

[1] Singh GAP, Gupta P (2018) Performance analysis of various machine learning-based approaches for detection and classification of lung cancer in humans. Neural Comput Appl. https://doi.org/10.1007/s00521-018-3518-x

[2] Dubey, S.R., Roy, S.K., Chakraborty, S. et al. (2020) Local bit-plane decoded convolutional neural network features for biomedical image retrieval. Neural Comput \& Applic 32, 75397551. https://doi.org/10.1007/s00521-019-04279-6

[3] Cai, W., Kim, J., Feng, D.D. (2008) Content based medical image retrieval, In Biomedical Information Technology, pp. 83-113. Elsevier.

[4] Ojala T, Pietikainen M, Maenpaa T (2002) Multiresolution grayscale and rotation invariant texture classification with local binary patterns. IEEE Trans Pattern Anal Mach Intell 24(7): 971-987. https://doi.org/10.1109/tpami.2002.1017623

[5] Pietika"inen M, Hadid A, Zhao G, Ahonen T (2011) Computer vision using local binary patterns, vol 40. Springer, Berlin.

[6] Murala, S., Maheshwari, R.P. \& Balasubramanian, R. Directional Binary Wavelet Patterns for Biomedical Image Indexing and Retrieval. J Med Syst 36, 2865-2879 (2012). https://doi.org/10.1007/s10916-011-9764-4

[7] S. Murala, R. P. Maheshwari and R. Balasubramanian, "Local Tetra Patterns: A New Feature Descriptor for Content-Based Image Retrieval," in IEEE Transactions on Image Processing, vol. 21, no. 5, pp. 2874-2886, May 2012, https://doi.org/10.1109/tip.2012.2188809

[8] Subrahmanyam Murala, Q.M. Jonathan Wu, (2013) Local ternary co-occurrence patterns: A new feature descriptor for MRI and CT image retrieval, Neurocomputing, Volume 119, Pages 399-412, ISSN 0925-2312, https://doi.org/10.1016/j.neucom.2013.03.018

[9] Subrahmanyam Murala, Q.M. Jonathan Wu, MRI and CT image indexing and retrieval using local mesh peak valley edge patterns, Signal Processing: Image Communication, Volume 29, Issue 3, 2014, Pages 400-409, ISSN 0923-5965, https://doi.org/10.1016/j.image. 2013.12.002

[10] Dubey, S.R., Singh, S.K. and Singh, R.K. (2016), Novel local bit-plane dissimilarity pattern for computed tomography image retrieval. Electron. Lett., 52: 1290- 1292. https://doi.org/ $\underline{10.1049 / \mathrm{el} .2016 .1206}$

[11] S. R. Dubey, S. K. Singh and R. K. Singh, "Local Diagonal Extrema Pattern: A New and Efficient Feature Descriptor for CT Image Retrieval," in IEEE Signal Processing Letters, vol. 22, no. 9, pp. 1215-1219, Sept. 2015, https://doi.org/10.1109/lsp.2015.2392623

[12] S. R. Dubey, S. K. Singh and R. K. Singh, "Local Wavelet Pattern: A New Feature Descriptor for Image Retrieval in Medical CT Databases," in IEEE Transactions on Image Processing, vol. 24, no. 12, pp. 5892-5903, Dec. 2015, https://doi.org/10.1109/tip. $\underline{2015.2493446}$

[13] Gonde A.B., Murala S., Vipparthi S.K., Maheshwari R., Balasubramanian R. (2017) 3D Local Transform Patterns: A New Feature Descriptor for Image Retrieval. In: Raman B., Kumar S., Roy P., Sen D. (eds) Proceedings of International Conference on Computer Vision and Image Processing. Advances in Intelligent Systems and Computing, vol 460. Springer, Singapore. https://doi.org/10.1007/978-981-10-2107-7_45

[14] Kaur, T., B. S. Saini, and S. Gupta. 2018. A novel feature selection method for brain tumor MR image classification based on the Fisher criterion and parameter-free Bat optimization. Neural Computing and Applications 29 (8):193-206. https://doi.org/10.1007/s00521-017$\underline{2869-z}$ 
Paper-A New Optimized Hybrid Local Lifting Wavelet Co-occurrence Texture Pattern for Content...

[15] Zhang, L., K. Mistry, C. P. Lim, and S. C. Neoh. 2018. Feature selection using firefly optimization for classification and regression models. Decision Support Systems 106:64-85. https://doi.org/10.1016/j.dss.2017.12.001

[16] Hu, L., W. Gao, K. Zhao, P. Zhang, and F. Wang. 2018. Feature selection considering two types of feature relevancy and feature interdependency. Expert Systems with Applications 93:423-34. https://doi.org/10.1016/j.eswa.2017.10.016

[17] Sayed, S., M. Nassef, A. Badr, and I. Farag. 2019. A Nested Genetic Algorithm for feature selection in high-dimensional cancer Microarray datasets. Expert Systems with Applications 121:233-43. https://doi.org/10.1016/j.eswa.2018.12.022

[18] Emary, E., H. M. Zawbaa, and A. E. Hassanien. 2016. Binary grey wolf optimization approaches for feature selection. Neurocomputing 172:371-81. https://doi.org/10.1016/j. neucom.2015.06.083

[19] Faramarzi, A., M. Heidarinejad, B. Stephens, and S. Mirjalili. 2020. Equilibrium optimizer: A novel optimization algorithm. Knowledge-Based Systems 191:105190. https://doi.org/10. $\underline{1016 / j . k n o s y s .2019 .105190}$

[20] Kashef, S., and H. Nezamabadi-pour. 2015. An advanced ACO algorithm for feature subset selection. Neurocomputing 147:271-79. doi:10.1016/j.neucom.2014.06.067. 26. Alex Stark J, “Adaptive image contrast enhancement using generalizations of histogram equalization", IEEE Transactions on Image Processing, VOL. 9, 2000. https://doi.org/10.1016/j.neucom. 2014.06.067

[21] Elisabeta Antonia Haller, “Adaptive histogram equalization in GIS”, Mathematics and Computer Science Series, Volume 38(1), Pages $100\{104,2011$.

[22] T. Ojala, M. Pietikainen and T. Maenpaa, "Multiresolution gray-scale and rotation invariant texture classification with local binary patterns," in IEEE Transactions on Pattern Analysis and Machine Intelligence, vol. 24, no. 7, pp. 971-987, July 2002, https://doi.org/10.1109/ tpami.2002.1017623

[23] B. Zhang, Y. Gao, S. Zhao, and J. Liu, "Local derivative pattern versus local binary pattern: Face recognition with higher-order local pattern descriptor," IEEE Trans. Image Process., vol. 19, no. 2, pp. 533-544, Feb. 2010. https://doi.org/10.1109/tip.2009.2035882

[24] X. Tan and B. Triggs, "Enhanced local texture feature sets for face recognition under difficult lighting conditions," IEEE Trans. Image Process., vol. 19, no. 6, pp. 1635-1650, Jun. 2010. https://doi.org/10.1109/tip.2010.2042645

[25] Z. Guo, L. Zhang, and D. Zhang, "Rotation invariant texture classification using LBP variance with global matching," Pattern Recogn., vol. 43, no. 3, pp. 706-719, Mar. 2010. https://doi.org/10.1016/j.patcog.2009.08.017

[26] Zhao, S., Gao, Y., and Zhang, B.: 'Sobel-LBP'. IEEE Int. Conf. Image Processing., San Diego, CA, USA, 2008, pp. 2144-2147 33. Jabeed, T., Kabir, M.H., and Chae, O.: 'Robust facial expression recognition based on local directional pattern', ETRI J., 2010, 32, pp. 784794. https://doi.org/10.4218/etrij.10.1510.0132

[27] ] Lambros S. Athanasiou, Dimitrios I. Fotiadis, Lampros K. Michalis, 4 - Plaque Characterization Methods Using Intravascular Ultrasound Imaging, Editor(s): Lambros S. Athanasiou, Dimitrios I. Fotiadis, Lampros K. Michalis, Atherosclerotic Plaque Characterization Methods Based on Coronary Imaging, Academic Press, 2017, Pages 71-94, ISBN 9780128047347, https://doi.org/10.1016/b978-0-12-804734-7.00004-x

[28] G. Bindu Madhavi, V. Vijaya Kumar, K. Sasidhar. The complete rank position matrix for content based image retrieval. JCR. 2020; 7(18): 997-1015.

[29] Lo P, Van Ginneken B, Reinhardt JM, Yavarna T, De Jong PA, Irving B, De Bruijne M (2012) Extraction of airways from CT (EXACT'09). IEEE Trans Medical Imaging 31(11):2093-2107. https://doi.org/10.1109/tmi.2012.2209674 
Paper-A New Optimized Hybrid Local Lifting Wavelet Co-occurrence Texture Pattern for Content...

[30] Clark K, Vendt B, Smith K, Freymann J, Kirby J, Koppel P, Moore S, Phillips S, Maffitt D, Pringle M, Tarbox L, Prior F (2013) The Cancer Imaging Archive (TCIA): maintaining and operating a public information repository. Journal of Digital Imaging 26(6):1045-1057. https://doi.org/10.1007/s10278-013-9622-7

[31] OASIS-MRI image database. http://www.oasis-brains.org/. Accessed 6 July 2016.

Tan X, Triggs B (2010) Enhanced local texture feature sets for face recognition under difficult lighting conditions. IEEE Trans Image Process 19(6):1635-1650. https://doi.org/10. 1109/tip.2010.2042645

[33] Zhang B, Gao Y, Zhao S, Liu J (2010) Local derivative pattern versus local binary pattern: face recognition with high-order local pattern descriptor. IEEE Trans Image Process 19(2):533-544. https://doi.org/10.1109/tip.2009.2035882

[34] Murala S, Wu Q (2014) Local mesh patterns versus local binary patterns: biomedical image indexing and retrieval. IEEE Journal of Biomedical and Health Informatics 18(3):929-938. https://doi.org/10.1109/jbhi.2013.2288522

[35] Murala S, Wu QJ (2015) Spherical symmetric 3D local ternary patterns for natural, texture and biomedical image indexing and retrieval. Neurocomputing 149, pp:1502-1514. https://doi.org/10.1016/j.neucom.2014.08.042

[36] Lan R, Zhou Y (2017) Medical image retrieval via histogram of compressed scattering coefficients. IEEE J Biomed Health Inform 21:1338-1346. https://doi.org/10.1109/jbhi.2016. $\underline{2623840}$

[37] Shinde A, Rahulkar A, Patil C (2019) Biomedical image indexing and retrieval based on new efficient hybrid approach using directional decomposition and a novel local directional frequency encoded pattern: a post feature descriptor. Multimed Tools Appl 78:2348923519. https://doi.org/10.1007/s11042-019-7697-y

[38] Deep G, Kaur L, Gupta S (2016) Directional local ternary quantized extrema pattern: A new descriptor for biomedical image indexing and retrieval. Elsevier. Engineering Science and Technology, an International Journal 19(4):1895-1909. https://doi.org/10.1016/j.jestch. 2016.05.006

[39] Shinde AA, Rahulkar AD, Patil CY (2017) Local Neighboring Binary Pattern: A New Feature Descriptor for Biomedical Image Indexing and Retrieval. Signal and Image Processing, ICSIP, pp: 154-159. https://doi.org/10.1109/siprocess.2017.8124524

[40] Shinde AA, Rahulkar AD, Patil CY (2017) Fast discrete curvelet transform-based anisotropic feature extraction for biomedical image indexing and retrieval. International Journal of Multimedia Information Retrieval, Springer 6 (4):281-288. https://doi.org/ $10.1007 / \mathrm{s} 13735-017-0132-0$

[41] Vandana Kate, Pragya Shukla (2021) Breast Cancer Image Multi-Classification Using Random Patch Aggregation and Depth-Wise Convolution based Deep-Net Model, iJOE - Vol. 17, No. 01: 83-100,https://doi.org/10.3991/ijoe.v17i01.18513

[42] Archana Kalidindi, Prasanna Lakshmi Kompalli, Sairam Bandi, Sri Raagh Rao Anugu (2021), iJOE - Vol. 17, No. 01: 51-62, https://doi.org/10.3991/ijoe.v17i01.18565

\section{$7 \quad$ Authors}

R. Varaprasada Rao is pursuing Ph.D. in the department of ECE at JNTUA, Ananthapuramu, Andhra Pradesh, India. His research interest includes Deep learning, Machine learning. Published many research articles in various international journals and conferences. 
Paper-A New Optimized Hybrid Local Lifting Wavelet Co-occurrence Texture Pattern for Content..

T. Jaya Chandra Prasad is working as a professor in the department of ECE at Rajeev Gandhi Memorial College of Engineering and Technology, Nandyal, Andhra Pradesh, India (Email: jp.talari@gmail.com).

Article submitted 2021-07-04. Resubmitted 2021-08-03. Final acceptance 2021-08-05. Final version published as submitted by the authors. 